\title{
The Third Catalogue of Nearby Stars - Results and Conclusions
}

\author{
Hartmut Jahreiß, Wilhelm Gliese \\ Astronomisches Rechen-Institut \\ Mönchhofstr 12-14 \\ D-6900 Heidelberg \\ Germany
}

\begin{abstract}
The nearby stars constitute the statistically most complete and therefore most representative sample of stars in our Galaxy. Only this sample allows a direct investigation of luminosity function and kinematics of low-mass stars.

A study of the Third Catalogue of Nearby Stars reveals a considerable increase - not only in quantity but also in quality - in our knowledge of the immediate solar neighbourhood, and reflects very nicely the progress achieved during the last decades - especially in astrometry and photometry. Expectations for the near future (HIPPARCOS) and desiderata for further astrometric and photometric work will be discussed.
\end{abstract}

\section{Introduction}

A preliminary version of the Third Catalogue of Nearby Stars (CNS3) became recently available on the CD-ROM Selected Astronomical Catalogs Volume 1 published by the Astronomical Data Center (NASA). The final version which is in progress will certainly not change the results reported in this paper. The CNS3 a compilation of astrometric, photometric and spectroscopic data - aims to list all known stars within 25 parsec of the Sun.

The basic data are the trigonometric parallaxes originating from a preliminary version of the forthcoming General Catalogue of Trigonometric Parallaxes (YPC) (van Altena et al. 1992). With these parallaxes new colour-magnitude calibrations (Jahreiß and Gliese, 1989) were carried out for various broad-band colours especially suited for red dwarf stars. These relations served as additional distance estimators, often in combination with likewise determined new spectroscopic parallaxes. Also Strœmgren photometry provided useful distances in particular for early-type stars having not seldom dubious trigonometric parallaxes.

Contrary to the CNS2 (Gliese, 1969) trigonometric and photometric or spectroscopic parallaxes were not combined. The resulting parallax is either the trigonometric parallax or the photometric (spectroscopic) parallax depending on the size of the parallax errors.

Within the last decade more than 2200 probable or suspected nearby objects were included in the working file which contains also all stars of former editions 
TABLE I

Statistics of the entire working file

\begin{tabular}{lrrrr}
\hline source & limit & all stars & CNS3 & removed \\
\hline Gliese (1969) & $22 \mathrm{pc}$ & 1913 & 1746 & 167 \\
Gliese \& Jahreiß (1979) & $22 \mathrm{pc}$ & 506 & 384 & 122 \\
${\text { Woolley et al. }(1970)^{*}}^{*}$ & $25 \mathrm{pc}$ & 410 & 285 & 125 \\
New data & $25 \mathrm{pc}$ & 2249 & 1388 & 861 \\
all & $25 \mathrm{pc}$ & 5078 & 3803 & 1275 \\
\hline
\end{tabular}

* only stars not in Gliese (1969) or Gliese and Jahreiß (1979)

even if modern observations proved that they are definitively beyond our distance limit of $25 \mathrm{pc}$. Table I gives the relevant figures in more detail and illustrates that about ten to twenty per cent of formerly considered nearby stars had now to be removed on the basis of more reliable distance determinations. This percentage is much higher in the last group New data, because every star was incorporated whenever there was the slightest indication that it may be nearby. This strategy should facilitate the search for additional information. At the very end only those stars entered into the catalogue for which the distance estimators fulfilled certain stringent conditions.

In the final step more than 2500 objects were rejected, so that the present version of the CNS3 contains 3803 stars: 2637 stars with trigonometric and 1165 stars having only photometric (and/or spectroscopic) parallaxes. For 734 stars the trigonometric parallax was replaced by a better photometric one. The CNS3 lists every star with $\pi_{t} \geq 0$." 0390 , even though it may be evident from photometry or for other reasons that the star is more distant. This applies very probably to 505 catalogue entries. On the other hand, also 224 stars were included with $\pi_{t}<0$. 0390 for which photometry and/or spectroscopy indicate a smaller distance. However, one has to keep in mind that such distances may be falsified by undetected companions. Consequently, almost $20 \%$ of the stars in CNS3 have still questionable distances. All these objects were marked not only to warn potential users, but also to stimulate additional observations.

\section{Content and Accuracy}

Figure 1 presents a colour-magnitude diagram of the 1090 stars with good trigonometric parallaxes $(\Delta \pi / \pi<0.14)$. It describes best the various types of objects contained in CNS3.

The average mean error in $M_{V}$ amounts to $\pm 0 m^{m} 33$. For $96 \%$ of the stars it should still be better than $\pm 0^{m} 75$ - a considerable progress compared to the $66 \%$ in 1957, when merely trigonometric and spectroscopic parallaxes were available. The average parallax error of the stars within 20 pc decreased from 0.014 in 1957 to 0.011 in 1969 and 0.008 in 1991 . Yet, this improvement, is obviously due to the fact that within the last decades trigonometric parallax work concentrated more and more on suspected nearby objects. Therefore, many low-luminosity stars with 


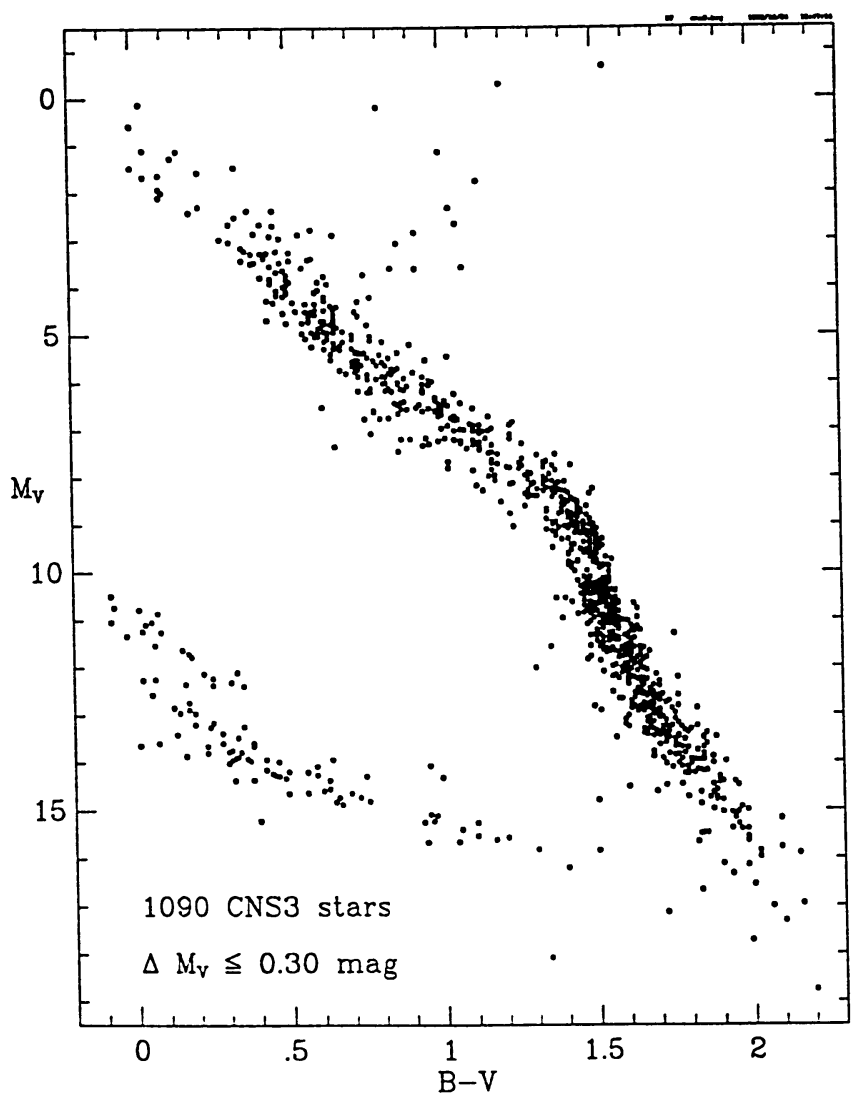

Fig. 1. Colour-Magnitude diagram of 1090 stars within $25 \mathrm{pc}$ having trigonometric parallaxes with $\Delta \pi / \pi<0.14$

high-accuracy $\pi_{t}$ (mainly from USNO, etc.) could be added. Whereas, for bright star there was almost no progress. They often have poor $\pi_{t}$ determined in the first half of our century. Luckily, HIPPARCOS will very soon change this situation completely.

A comparison of the distances in CNS2 with the new ones reveals no significant systematic differences. Only a slight tendency to smaller parallaxes seems to be present for the more distant stars. This may be due to the well known effect that in a volume limited sample parallaxes with positive errors are prefered and therefore on the average too large. Consequently, modern parallaxes with higher accuracy should diminish this effect.

Positions and proper motions are for epoch and equinox B1950. The CDA (Jahreiß, 1989) with a typical proper motion error $\Delta \mu=0.006$ contributes $67 \%$. For another $25 \%$ photographically determined proper motions (LHS, NLTT, Giclas) were available with typical errors of $\Delta \mu=0.014$ if machine-measured, and $\Delta \mu=0$. 025 or larger if hand-blinked. New measurements are in progress for 50 
TABLE II

Catalogues of Nearby Stars $1957-1991$

\begin{tabular}{rrrrr}
\hline distance & 1957 & 1969 & 1991 & $* /\left[\mathrm{pc}^{3}\right]$ \\
\hline $0-5 \mathrm{pc}$ & 52 & 54 & 61 & 0.12 \\
$5-10 \mathrm{pc}$ & 179 & 207 & 265 & 0.07 \\
$10-20 \mathrm{pc}$ & 863 & 918 & 1795 & 0.06 \\
$20-25 \mathrm{pc}$ & & & 1282 & 0.04 \\
total & 1094 & 1890 & 3803 & 0.06 \\
\hline
\end{tabular}

stars with unknown proper motions.

Radial velocities could be found for 1945 stars. Already $51 \%$ of these radial velocities were photoelectrically determined, thus improving the resulting space velocity components.

Weighted mean values of UBV photometry in the Johnson system and R-I in Kron's system are given as far as available to us. There are still 838 stars without B-V, 1380 stars without U-B, and 1183 stars without R-I colour indices.

The CNS3 contains 552 systems with althogether 1091 components, not counted are 195 spectroscopic binaries (63 with known orbits), 45 suspected SB's, and 43 stars with variable radial velocity.

\section{Completeness}

The faintest star in Figure 1 is vB 10 with $M_{V}=18^{m} 76$. Still fainter is LHS 2924 with $M_{V}=19^{m} 63$. The $25 \mathrm{pc}$ limit corresponds to a distance modul of $M-m=$ $-1^{m} 99$. Therefore, the complete sky down to $m_{V} \sim 22^{m}$ should be surveyed, in order to detect all nearby stars. This is a task which is - with present means practically not feasible. Consequently, the CNS3 is highly incomplete. This becames obvious from Table II showing the progress achieved during the past decades, but also - in the last column - the still present steep decrease of the number density with increasing distance.

Though, the number of stars increased significantly, e. g. in the interval from 10 to 20 pc it almost doubled since 1969 , the number density itself decreases very rapidly from $0.12 \mathrm{stars} / p c^{3}$ within $5 \mathrm{pc}$ to $0.04 \mathrm{stars} / p c^{3}$ for the outermost shell from $20 \mathrm{pc}$ to $25 \mathrm{pc}$. One may even question if the innermost $5 \mathrm{pc}$ sphere is complete. Since 1982 no additional single star was found within 5 pc. But, of course, one cannot exclude that there are still some undetected stars within this limit.

In Table II only resolved binaries were counted. At present there are 70 objects known within $5 \mathrm{pc}$ if one takes into account also unresolved spectroscopic and/or astrometric binaries. This yields a relative binary frequency of $51 \%$, which is much more than the $35 \%$ for the entire CNS3. The relative binary frequency amounts even to $58 \%$ according to the results of an extensive search for faint companions around the nearest stars north of $\delta=30^{\circ}$ which was carried out with the aid of high-precision radial velocity measurements and infrared speckle interferometric 


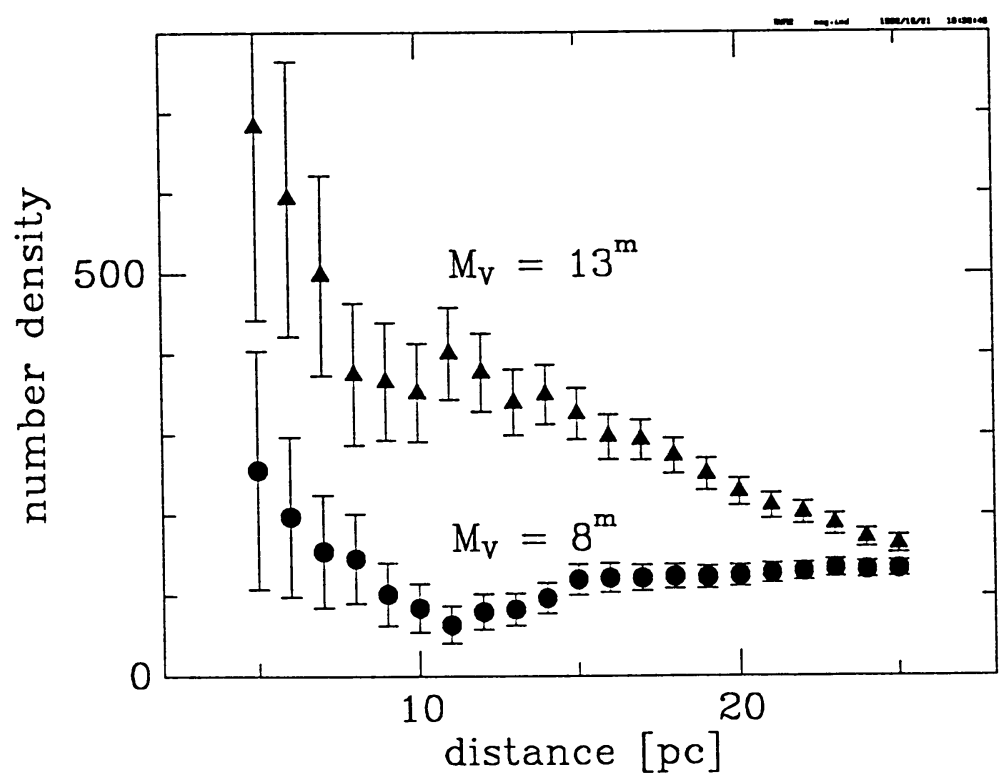

Fig. 2. Normalized number density of stars versus distance from the Sun for $M_{V}=8^{m}$ (filled circle) and $M_{V}=13^{m}$ (filled triangle). The errors are Poisson-errors.

observations (Henry and McCarthy, 1990). All companions with masses above 80 Jupiters should have been detected. A similar observing program recently started for the far southern stars may detect additional companions.

The 70 objects within $5 \mathrm{pc}$ lead to a mean distance of $2.2 \mathrm{pc}$ between the stars. Proxima Centauri, however, is only $1.3 \mathrm{pc}$ away from the Sun. Is this a further hint for undetected objects?

Of course, the completeness depends of the luminosity of the stars. A more detailed analysis shows that the CNS3 is statistically complete for stars with $M_{V}<$ $9^{m}$. This becomes evident in Figure 2 where the number density of the stars (normalized to a volume of $20 \mathrm{pc}$ radius) is plotted against increasing distances from the Sun. For bright stars $M_{V}=8^{m}$ the number density is rather constant out to $25 \mathrm{pc}$, whereas it decreases very rapidly for the faint stars $M_{V}=13^{m}$.

\section{Conclusions}

Within the last decades extensive proper motion surveys and spectroscopic surveys were carried out. A large amount of high-accuracy trigonometric parallaxes for faint stars became available allowing improved calibrations of colour-luminosity relations and therefore in combination with subsequent photoelectric photometry a more effective exploitation of the above mentioned surveys. All this resulted in a considerable increase of known nearby stars. Nevertheless, the CNS3 is not only incomplete - the widely accepted luminosity function from Wielen et al. (1983) predicts more than 7700 stars within $25 \mathrm{pc}$, but also inhomogeneous with respect 
to the information available for individual stars. Now, the incompleteness cannot be avoided with present means, and this is also not necessary. A growing possibility to create well defined representative subsamples will be sufficient for reliable investigations of the different populations in the solar neighbourhood with respect to spatial distribution, luminosity function and kinematics.

Considerable efforts are required regarding the very faint stars. Up to now they were primarily found among the high-proper motion stars, and it is still the most effective way. With Luyten's photographic proper motion surveys (LHS, NLTT) the search can be extended to $m_{p g}=21^{m}$. The drawback of such a search is, however, the resulting bias to high-velocity stars. But, spectral surveys for red dwarf stars avoiding this bias are presently limited to $m_{V} \sim 11^{m}$.

For the near future one can expect from the astrometric satellite HIPPARCOS not only much better positions, proper motions and trigonometric parallaxes, but also much more additional information on duplicity and variability of the stars. Yet, HIPPARCOS will only observe stars brighter than $\left(m_{B} \sim 12^{m} 5\right)$ as far as they were selected for the Input Catalogue.

With respect to the fainter stars several digitized sky surveys with limits of $m_{p g} \sim 21^{m}$ or even fainter will soon become available, and may provide at least new nearby candidates. A promising proposal to detect the low-velocity portion of the faint nearby stars is by subsequent optical analysis of X-ray sources found by ROSAT (Fleming, 1988, 1992).

\section{References}

Fleming, T. A. (1988) Optical Analysis of an X-ray selected Sample of stars. PASP 100, p. 1186

Fleming, T. A. (1992) private communication

Gliese, W. (1957) Katalog der Sterne näher als 20 Parsek für 1950.0 Mitteilungen des Astronomischen Rechen-Instituts Serie A Nr. 8

Gliese, W. (1969) Catalogue of Nearby Stars. Edition 1969 Veröffentlichungen des Astronomischen Rechen-Instituts Heidelberg Nr. 22

Gliese, W. and Jahreiß, H. (1979) Nearby Star Data Published 1969-1978, Astron. Astrophys. Suppl. 38, p. 423

Henry, T. J. and McCarthy, Jr. D. W. (1990) A Systematic Search for Brown Dwarfs Orbiting Nearby Stars. Astrophys. J. 350, p. 334

Jahreiß, H. (1989) The Compilation of Astrometric Data, in The HIPPARCOS Mission. PreLaunch Status. Vol II: The Input Catalogue. M. A. C. Perryman (Scientific Coordinator), European Space Agency, Paris, ESA SP-1111, p. 115

Jahreiß, H. and Gliese, W. (1989) The Third Catalogue of Nearby Stars. II. Applied Methods and Use. in Star Catalogues: A Centennial Tribute to A. N. Vyssotsky, Proc. Meeting IAU Comm. 24, Baltimore, MD, USA, eds. A. G. D. Philip, A. R. Upgren, L. Davis Fress, Schenectady, N. Y. p. 11

van Altena, W. F., Lee, J. T., and Hoffleit, D. (1992) General Catalogue of Trigonometric Stellar Parallaxes. Yale Univ. Obs., in preparation

Wielen, R., Jahreiß, H., and Krüger, R. (1983) The Determination of the Luminosity Function of Nearby Stars. in IAU Coll. 76, The Nearby Stars and the Stellar Luminosity Function. eds. A. G. D. Philip, A. R. Upgren, L. Davis Press, Schenectady, N.Y. p. 163

Woolley R., Epps, E. A., Penston, M. J. and Pocock, S. B. (1970) Catalogue of Stars within twenty-five parsec of the Sun. Royal Obs. Annals No. 5 